\title{
Field Feasibility and Acceptability Testing of Action-Based Psychosocial Reconciliation Approach in a Post-Genocide Rural Community in Rwanda: A Study Protocol
}

\author{
Masahiro Minami ${ }^{1^{*}}$ \\ ${ }^{1}$ Faculty of Education, Simon Fraser University, 250-13450 102 Avenue, Surrey, British Columbia, \\ Canada V3T 0A3
}

\begin{abstract}
Background: Interpersonal/psychosocial reconciliation is highly prioritized in post-Genocide Rwanda. Despite the need, empirically sound strategies have been extremely scarce. The proposed study is a segment of a broader services-research effort to develop, evaluate, and implement a novel and empirically supported interpersonal/psychosocial reconciliation approach termed Action-Based Psychosocial Reconciliation Approach (ABPRA), that is authentically founded on Rwandan people's lived experiences of reconciliation.

Methods/Design: The proposed study consists of two major steps. The purpose of step 1 is to develop and empirically validate a set of outcome measures, termed the psychosocial reconciliation impact scales module (PRISM) to assess beneficial impacts native to ABPRA. We will employ hermeneutic phenomenological analysis (van Manen, 2016) of pilot interview data to generate item pool. The purpose of step 2 is to field-test the delivery of ABPRA in Rwanda to evaluate the feasibility and acceptability of the intervention, exploring and illuminating potential procedural uncertainties in conducting a larger-scale evaluation of ABPRA. We will follow the guidance on pilot study by Thabane et al. (2010).

Discussion: The study is an essential step to advance the project to a full-scale experimental evaluation of ABPRA. The project holds the possibility of making available and accessible, an empirically supported and meaningful approach to conflict resolution, genocide/war prevention and peacebuilding in Rwanda and other war/conflict-affected regions around the globe.
\end{abstract}

Keywords: psychosocial reconciliation, feasibility and piloting, Morita therapy, contact theory, the 1994 Genocide against the Tutsi

\footnotetext{
* Correspondence concerning this article should be addressed to Masahiro Minami, Faculty of Education, Simon Fraser University, 250-13450 102 Avenue, Surrey, British Columbia, Canada V3T 0A3. Email: mminamia@sfu.ca
} 


\section{Background}

\subsection{Study context and background}

In April of 1994, the Genocide against the Tutsi took place in Rwanda. UNICEF (Chauvin, Mugaji, \& Comlavi, 1998; Dyregrov, Gupta, Gjestad, \& Mukanoheli, 2000) reported that between April and July, approximately 800,000 to 1 million people of Tutsi ethnic background were systematically murdered by Hutu extremists, the Interahamwe. Subsequently, in 2003, a presidential decree was released to pardon and reintegrate genocide perpetrators/prisoners back into their community. The 1994 Genocide was characterized as an "intimate genocide" in which families, relatives, friends, and neighbours sharing the same village/community turned to kill each other (Staub, Pearlman, Gubin, \& Hagengimana, 2005). The 2003 release of prisoners led to the rare circumstance (in the context of post-war restitution) in which genocide survivors would have to live 'side by side' with the returning perpetrators to share the same home community (McGarty, 2014).

Interpersonal/psychosocial reconciliation for groups and individuals is highly prioritized, promoted, and urged as a way of achieving conflict resolution, prevention, and peacebuilding in rural communities in Rwanda today (National Unity and Reconciliation Commission, 2020). Despite the grave need, available research-informed strategies to foster interpersonal/psychosocial reconciliation have been extremely scarce. Among the very few is the seminal work undertaken by Drs. Staub and Perlman, employing a theory-driven approach to promote healing, unity, and reconciliation in Rwanda (Staub et al., 2005). Due to its methodological limitations in an experimental design, their studies left unanswered other research questions that could advance the field of interpersonal/psychosocial reconciliation and the effectiveness of currently available approaches: What is the nature and process of interpersonal reconciliation as experienced by the very survivors and perpetrators in Rwanda? What may be critically missing is our understanding of the very lived experiences of people in the process of reconciliation today and a reconciliation approach that is founded on these authentic experiences.

The proposed study is a segment of a broader services-research project that has been undertaken by the PI since 2011 in Rwanda. The goal of the project is to develop and make available a research-informed interpersonal/psychosocial reconciliation approach closely founded on Rwandan people's lived experiences of reconciliation to nurture authentic reconciliation, recovery, and growth in rural villages of Rwanda. In terms of scholarship, the proposed study is situated in the broader areas of (a) post-war/conflict psychosocial development strategies, (b) interpersonal and psychosocial reconciliation process and outcome, (c) peace and conflict studies, (d) war/conflict/genocide prevention, (e) forgiveness, (f) restorative justice and reparation approaches, (g) intergroup contact theory, (h) attitude and attitude change, (i) mental health services-research, and (j) implementation science. The project as a whole, is an application of mental health services research and implementation science strategies to aid postconflict/war reconciliation and psychosocial development.

\subsection{Development of Action-Based Psychosocial Reconciliation Approach (ABPRA)}

ABPRA (Minami, 2020) is a research-informed and practical approach developed by conducting a series of narrative literature reviews to model and empirically support micromechanisms facilitative of healing and reconciliation among conflicting parties. Healing mechanisms are grounded in the therapeutic principles of Japanese Morita therapy (Morita, 
1974). Seminal works in the area of post-conflict reconciliation suggested that the essence of reconciliation is in a mutual attitude change (Bar-Tal, 2000; Staub, 2008; Staub et al., 2005). Therefore, mechanisms facilitative of positive attitude change between conflicting parties are also architected in the ABPRA and founded on principles of intergroup contact theory (Allport, 1954; Amir, 1969; Brewer \& Kramer, 1985; Pettigrew, 1997, 1998; Pettigrew \& Tropp, 2006). Harmonised together, ABPRA is a practical synthesis and translation of Japanese Morita therapy and intergroup contact theory into an interpersonal reconciliation approach.

ABPRA (Minami, 2020) was developed as an alternative approach to forgiveness (Hamber, 2007; Worthington, 2005, 2006) -based reconciliation counselling (FBRC) - popularly practised in post-genocide Rwandan villages (Hinson, 2009). In the forgiveness-based approach, perpetrators ask survivors to forGIVE (Hinson, 2009). Forgiveness-based approaches, however, are limited in cases where survivors are unable to forgive wholeheartedly. ABPRA was developed to welcome survivors and perpetrators acknowledge, recognize, and respect when forgiveness is not possible and invites survivors and perpetrators to explore instead possible ways to take purposeful, practical, and productive actions/interactions (e.g., collaborative labour) for the service of survivors. In ABPRA, perpetrators ask survivors if they would consider RECEIVING service from perpetrators as concrete acts (behavioural expressions) of their apology. Survivors are then given ample time to consider. If they choose to do so, perpetrators and survivors work together in the service of survivors for 2 hours per day, once per week for a total of 8 sessions. ABPRA aims to reorient the reconciling parties' efforts and focus away from the futile attempt to verbally seek ('control') forgiveness, in favour of constructive action/interaction-taking. Previous participants of ABPRA nicknamed it ubwiyunge mubikorwa (reconciliation in action).

ABPRA (Minami, 2020) also features an intervention that departs from one that attempts to "induce" forgiveness and reconciliation, to one that acknowledges and honours the reality of varied experiences, including the inability to forgive. In cases where survivors agree to accept service, perpetrators offer their labour as an impetus to invite survivors to have new dyadic experiences of receiving help and support from the perpetrators. Then, the process of reconciliation continues in action and interaction, and the new dyadic experiences hold the potential to nurture desired outcomes (e.g., forgiveness, reconciliation) in the heart of survivors and perpetrators. In short, ABPRA nurtures, rather than attempts to effect, authentic and idiographic experiences of healing and interpersonal reconciliation.

\subsection{Field piloting of ABPRA}

Between 2011 and 2013, ABPRA (Minami, 2020) was implemented in two rural villages of Rwanda to qualitatively explore lived experiences of survivors and perpetrators engaged in the process of ABPRA. The pilot was conducted in partnership with Prison Fellowship Rwanda (PFR) and the Rwanda National Unity and Reconciliation Commission (NURC). Over the two years, a total of 8 dyads consisting of survivors and their direct perpetrators who lived in the same community volunteered to participate in ABPRA. Our research team collected over 7,200 minutes of qualitative interview data capturing participants' lived experiences in the ABPRA. The interview data were then transcribed in Kinyarwanda, translated to English and backtranslated to check translation accuracy and analysed by employing a thematic content analysis (Krippendorff, 2014). Five major themes emerged representing major beneficial effects (referred to as properties) of ABPRA: (a) healing, (b) attitude change, (c) reconciling, (d) relationship building and (e) psychosocial development (Minami, 2014, 2020). Field piloting of ABPRA was Social Science Protocols, February 2021, 1-12. 
completed with encouraging results for ABPRA, along with a rich pool of narrative data articulating authentic and idiographic processes of interpersonal/psychosocial reconciliation. Our two years of preliminary field research and data have enabled us thus far to develop (a) an ABPRA intervention protocol along with its full micro-mechanisms of change modelling, (b) an ABPRA reconciler training manual, and (c) ABPRA intervention fidelity check sheets. The pilot data continue to serve as an essential empirical foundation for the proposed study and future evaluation.

\subsection{Proposed study}

The ultimate goal of the project in Rwanda is to develop ABPRA into an evidence-based approach to interpersonal/psychosocial reconciliation which can be made available and accessible to all Rwandans. Generating rigorous and robust data supporting the efficacy of a complex intervention that can be openly accessible and transparently appraisable by anyone interested is an essential pre-requisite to any evidence-based intervention (Craig et al., 2006) and an integral objective of this project.

The MRC Guidance on Developing and evaluating complex interventions (Craig et al., 2006) sets forth 4 recommended phases of (a) development, (b) feasibility/piloting, (c) evaluation, and (d) implementation for the development of complex interventions. The proposed study consists of two steps, encompassing the initial two phases of the guidance and serves as an essential bridge between the pilot and future large-scale evaluation studies. The MRC guidance recommends the development of any complex intervention to undertake the step examining its feasibility/piloting before proceeding to a costly large-scale evaluation (Craig et al., 2006; Thabane et al., 2010).

Step 1 of the proposed study (corresponding to the phase 1 development of the MRC guidance) prepares for the larger-scale evaluation of ABPRA by developing and validating an outcome measure optimal in assessing unique beneficial impacts of ABPRA. Step 2 of the proposed study (phase 2 feasibility/piloting) carries out the feasibility and acceptability testing of ABPRA in the actual field setting in Rwanda, exploring and understanding potential procedural uncertainties in conducting a larger-scale evaluation of ABPRA. This step of the study adheres to the exemplary guidance on piloting studies set forth by Thabane et al. (2010).

\section{Methods/Design}

\subsection{Step 1: Development and preliminary validation of the Psychosocial Reconciliation Impact Scales Module (PRISM)}

The purpose of step 1 of the study is to develop and empirically validate a set of outcome measures containing five sub-scales, termed the psychosocial reconciliation impact scales module (PRISM) to assess unique beneficial impacts native to ABPRA. Standard guidelines for test construction (Clark \& Watson, 1998; DeVillis, 2017; Nunnally \& Bernstein, 1994; McIntire \& Miller, 2000) and item writing (Netemeyer, Bearden \& Sharma, 2003; Spector, 1992; Pedhazur \& Schmelkin, 1991) will be followed throughout the development of the PRISM. Key activities of this step are as follows:

\section{Ethics approval status}

Ethics approval for step 1 has been granted by the Simon Fraser University (Canada) Research Ethics Board. 


\section{Hermeneutic phenomenological analysis (H.P.A.) of pilot data}

We begin this step by conducting a secondary reanalysis of the pilot interview data, collected from the lived experiences of survivors and perpetrators engaged in ABPRA, employing the hermeneutic phenomenological analysis (HPA) (van Manen, 2016). HPA is considered optimal in analysing text data and revealing key themes, along with the associated narratives that are representative of beneficial impacts of ABPRA as experienced by the very participants. The emerged themes will be compared to the original five major domains of beneficial impact reported by the pilot. Depending on the outcomes of the HPA, we may update the pilot themes. The final themes will serve as the domains of the PRISM, and the associated narratives will be re-phrased to serve as the scale items.

\section{Construct explication and domain specifications}

Informed by the pilot data and the HPA, we will employ a construct explication approach (Murphy \& Davidshofer, 1998; Cronbach \& Meehl, 1955; Cronbach, 1971; Messick, 1989) to define operationally and fully explicate each of the emerged themes as a construct domain to be measured by each subscale.

\section{Item writing/development}

Based on the pilot, we estimate 50 items in total for the final PRISM to be optimal in ensuring the feasibility of administration in the field (DeVellis, 2017; Nunnally \& Bernstein, 1994) and having desirable psychometric properties (Furr \& Bacharach, 2014). The initial pool of 150 items (three times the number of items to be included in the final product) will be developed based on the HPA, and tested for this step (Pett et al., 2003). The exact proportion of items allocated to each sub-scale corresponds to the exact proportion of meaning units emerged for each theme from the pilot study, namely a total item pool of 30 will be developed and tested for the (a) healing sub-scale, 60 for the (b) attitude change, 33 for the (c) reconciliation, 18 for the (d) relationship building, and 9 for the (e) psychosocial development subscales.

\section{Scale validation procedures}

Preliminary validation data collection will be conducted by employing an online survey tool (SFU Survey System). A confirmatory factor analysis (Gorsuch, 1983; 2014) (Maximum Likelihood Model; Joreskog, 1967, 1969; Long, 1983) with minimum $\mathrm{n}=300$ will test the presence of 5 scales: (a) healing, (b) attitude change, (c) reconciliation, (d) relationship building, and (e) psychosocial development. A posthoc exploratory factor analysis with principal component analysis (Gorsuch, 1983; 2014) will be performed to 'reduce' items in each sub-scale, and only items with higher factor loading will be selected (DeVellis, 2017; Pett et al., 2003; Floyd \& Widaman, 1995). We estimate the final number of items per subscale as 10 for (a) healing, 20 for (b) attitude change, 11 for (c) reconciliation, 6 for (d) relationship building, and 3 for (e) psychosocial development, reflecting the differential proportion of meaning units emerged for each theme. The final number of items depends on achieving aimed reliability $(\alpha \geq 0.90)$ (Cronbach, 1951) and other validity indicators (Cattell, 1966; Cliff, 1988; Humphreys et al., 1969; Pett et al., 2003).

\subsection{Step 2: Field feasibility and acceptability testing of the ABPRA}

The second step of the proposed study will be conducted in the Republic of Rwanda, in collaboration with PFR and NURC who have been key research partners of the PI since 2011. Social Science Protocols, February 2021, 1-12. 
The purpose of this subsequent step is to field-test the delivery of ABPRA in Rwanda in order to evaluate the feasibility and acceptability of the intervention, exploring and illuminating potential procedural uncertainties in conducting a larger-scale evaluation of ABPRA. The study also examines its expected reconciliation impact employing the PRISM. Specific objectives of step 2 include:

(a) training 15 previous participants of ABPRA as the new ABPRA reconcilers,

(b) training 4 ABPRA field researchers,

(c) conducting post-training session semi-structured interviews to explore all trainees' preliminary views on the feasibility and acceptability of ABPRA,

(d) conducting field implementation of ABPRA delivered by the newly trained ABPRA reconcilers,

(e) conducting a mixed-method evaluation of ABPRA by the newly trained ABPRA field researchers,

(f) conducting post-session interviews of the newly trained ABPRA reconcilers to explore their views on its acceptability and feasibility,

(g) conducting interviews of the newly trained ABPRA field researchers to explore their views on its acceptability and feasibility at the end of all session

\section{Ethics review applications}

Ethics review applications for step 2 are to be submitted to the Simon Fraser University Research Ethics Board and the Rwanda National Ethics Committee towards the completion of step 1 of the study in late 2021.

\section{Training of ABPRA reconcilers}

Upon obtaining ethics review approvals, the PI will conduct two types of training seminars in Rwanda in collaboration with local partners. First, a one-day training seminar (totalling 8 hours) will be conducted to train all 15 previous participants of ABPRA (consisting of survivors and their direct perpetrators from the pilot) as the new ABPRA reconcilers. We will employ a standardized ABPRA intervention protocol, a training manual, and an intervention fidelity check sheets for the training. We estimate requiring 8 reconcilers in total for the future large-scale evaluation. However, based on PI's field piloting experience, we anticipate possible attrition among reconcilers due to health and other concerns (e.g., deterioration of existing conditions such as HIV). Thus, taking these possibilities into account, we aim for training all 15 previous participants. The PI has maintained contact through annual visits, and they have expressed interests and desires to share their experience of ABPRA and help support their fellow Rwandans' journey of reconciliation. Our team believes that the very survivors and perpetrators who have nurtured their own processes of interpersonal reconciliation through ABPRA would be best suited to support others in same/similar positions.

The training seminar will be followed by the post-session semi-structured interviewing of the trainees to gather their perspectives on field feasibility and acceptability of the ABPRA as an interpersonal/psychosocial reconciliation approach. We plan on employing a hybrid phenomenological-thematic content analysis approach situated in the social constructionist paradigm (Gergen, 2001). Phenomenological methodology (with an interview method) (Langdridge, 2007; Crotty, 1998) is considered suitable for exploring 'experience' of an individual in an open-ended manner, while acknowledging that reliability, validity and generalizability are not the main priority parameters (Smith, Flowers, \& Larkin, 2009). This 
method fits well with the exploratory nature of this step. Thematic content analysis (Krippendorff, 2014) will be employed to analyze and categorize the interview data into (a) feasibility and (b) acceptability-related themes, outlined in Thabane et al. (2010). To ensure trustworthiness, the themes will be validated by the member check procedure with the trainees (Smith, Flowers, \& Larkin, 2009).

\section{Training of ABPRA field researchers}

Next, a two-day seminar (totalling 16 hours) will be conducted to train 4 ABPRA field researchers, employing a standardized ABPRA research protocol. The training seminar will begin with an overview of ABPRA, employing the ABPRA intervention protocol, the training manual, and the intervention fidelity check sheets. The second day of the training seminar will scope an overview of our research methodologies and methods, including practice sessions of interviewing skills through role-plays. The interview schedule for new participants will be adopted from our pilot study to explore participants' (a) affective (e.g., any feeling experienced) and (b) cognitive (e.g., any thoughts occurred) experiences during the ABPRA sessions. This training seminar will also be followed by the post-session semi-structured interview to explore trainees' thoughts and inputs pertinent to the field feasibility and acceptability of ABPRA. We will employ the same methodology and methods for data collection and analysis as were employed for the ABPRA reconciler training.

\section{Participant recruitment}

Concurrent with the two training seminars, the PI will engage in field recruiting of new participants in the village of Mbyo (coordinates specified: -2.222831, 30.157798), in collaboration with PFR. PFR has been supporting the community reconciliation process of Mbyo since 2005, where more than 63 houses were built for returning survivors and perpetrators to live in the same community. The PI's initial field piloting (2011-13) was also supported by PFR and participants from the Mbyo village. 13 of the 15 future ABPRA reconcilers also reside together in Mbyo. The proposed study will continue to be hosted by PFR and Mbyo to ensure maximum safety for all parties involved. A village-wide orientation meeting will be held to inform villagers of the study and recruit 4 new volunteer dyads. Dyads will consist of 4 new survivors and 4 new corresponding perpetrators. We have also developed an informed consent form for participants in both English and in the local language of Kinyarwanda.

\section{Implementation and data collection}

Upon obtaining informed consent from each participant, ABPRA will be implemented by the 8 newly trained ABPRA reconcilers. They will work in four survivor-perpetrator pairs with each pair matched with a newly recruited dyad (consisting of a new survivor and a perpetrator). As per ABPRA intervention protocol, each new reconciliation dyad will be asked to engage in joint labour in service of the survivor for 2 hours per day, once per week for a total of 8 sessions. ABPRA reconcilers will follow the intervention protocol and fidelity check sheet to ensure standardized delivery and intervention fidelity of the ABPRA. At the completion of each session, each one of the 4 newly trained ABPRA field researchers will (a) administer the PRISM, and (b) conduct a post-session semi-structured interview of the new participants to explore their lived experiences of ABPRA. The ABPRA reconcilers will not be involved in the post-session interview to circumvent potential intervener bias in the interviewing process. Ensuring the separate researchers will therefore ensure optimal impartiality in collecting data.

Social Science Protocols, February 2021, 1-12. 
At the end of each session, each of the 8 ABPRA reconcilers will also be interviewed by the field researchers to explore their views on feasibility and acceptability. We have decided to conduct this interview as we expect there would be differing issues of feasibility and acceptability emerging at the beginning, middle, and later stages of session progressions. Interviewing at the end of each session would allow us to get the sense of temporal factors relevant to program feasibility and acceptability. The field researchers will also keep field journals throughout the study to record any relevant feasibility and acceptability observations. The following Table 1 summarizes the types of data we expect to collect, proposed methods of analysis for each type of data, and their intended use.

Table 1. Types of data, methods of analysis, and their intended purposes.

\begin{tabular}{|c|c|c|}
\hline $\begin{array}{l}\text { Types of Data to be } \\
\text { Collected }\end{array}$ & $\begin{array}{l}\text { Proposed Methods of } \\
\text { Analysis }\end{array}$ & Intended Purpose \\
\hline $\begin{array}{l}\text { ABPRA reconcilers post- } \\
\text { training interview data }\end{array}$ & $\begin{array}{l}\text { Thematic content analysis } \\
\text { employing feasibility and } \\
\text { acceptability themes outlined } \\
\text { by Thabane et al. (2010) }\end{array}$ & $\begin{array}{l}\text { Exploring feasibility and } \\
\text { acceptability of procedures }\end{array}$ \\
\hline $\begin{array}{l}\text { ABPRA field researchers } \\
\text { post-training interview data }\end{array}$ & $\begin{array}{l}\text { Thematic content analysis } \\
\text { employing feasibility and } \\
\text { acceptability themes outlined } \\
\text { by Thabane et al. }\end{array}$ & $\begin{array}{l}\text { Exploring feasibility and } \\
\text { acceptability of procedures }\end{array}$ \\
\hline $\begin{array}{l}\text { PRISM data from each } \\
\text { ABPRA session }\end{array}$ & $\begin{array}{l}\text { Performing repeated } \\
\text { measures ANOVA } \\
\text { employing SPSS }\end{array}$ & $\begin{array}{l}\text { Evaluating beneficial impact of } \\
\text { ABPRA, and estimating } \\
\text { appropriate/feasible sample } \\
\text { sizes for the larger trial }\end{array}$ \\
\hline $\begin{array}{l}\text { Post-session semi-structured } \\
\text { interview data from new } \\
\text { participants }\end{array}$ & Thematic content analysis & $\begin{array}{l}\text { Exploring and examining } \\
\text { beneficial impact of ABPRA }\end{array}$ \\
\hline $\begin{array}{l}\text { Post-session semi-structured } \\
\text { interview data from new } \\
\text { ABPRA reconcilers }\end{array}$ & $\begin{array}{l}\text { Thematic content analysis } \\
\text { employing feasibility and } \\
\text { acceptability themes outlined } \\
\text { by Thabane et al. }\end{array}$ & $\begin{array}{l}\text { Exploring feasibility and } \\
\text { acceptability of procedures }\end{array}$ \\
\hline $\begin{array}{l}\text { Field journal collected from } \\
\text { the ABPRA field researchers }\end{array}$ & $\begin{array}{l}\text { Thematic content analysis } \\
\text { employing feasibility and } \\
\text { acceptability themes outlined } \\
\text { by Thabane et al. }\end{array}$ & $\begin{array}{l}\text { Exploring feasibility and } \\
\text { acceptability of procedures }\end{array}$ \\
\hline
\end{tabular}

\section{Data analyses plan}

The PRISM (pre-and post-intervention) data will be analyzed by employing repeated measures ANOVA, and, along with other data, be used to determine optimal/feasible sample size for future evaluation studies. The post-ABPRA session semi-structured interview data from the new participants will be translated into English and analyzed by employing thematic content analysis (TCA; Krippendorff, 2014) with no a-priori themes. The emergent themes will be compared with the five major themes reported by the pilot study (Minami, 2020) exploring their similarities and differences, and replicability of the beneficial impacts of the ABPRA. All Social Science Protocols, February 2021, 1-12. http://dx.doi.org/10.7565/ssp.v4.5199 
remaining qualitative data will also be analyzed via TCA, employing feasibility and acceptability themes outlined by Thabane et al. (2010).

\section{Discussion}

To date, no impact assessment measure has been developed exclusively from the lived experiences of people reconciling in post-genocide Rwandan communities. In contrast to existing measures that are theoretically derived (Staub et al., 2005; Stammel et al., 2015), the PRISM will be the first instrument 'empirically derived' from the authentic lived experiences and narratives of survivors and perpetrators engaged in the process of reconciliation through ABPRA. We expect that the PRISM will demonstrate (a) optimal fitness to assess/capture unique and novel beneficial impacts of ABPRA, (b) strong face/external/ecological/cross-cultural validity, (c) optimal construct representation of interpersonal/psychosocial reconciliation process in Rwanda, and (d) optimal accessibility and feasibility for its use in rural settings of Rwanda.

ABPRA is a distinct approach, harmonizing eastern (Japanese Morita Therapy) and western (contact theory) paradigms for conflict resolution, and transforms them into an interpersonal/psychosocial reconciliation approach that is practical, economical, productive/generative, and self-sustainable. These characteristics or properties of a psychosocial intervention are much desired, especially in the context of developing countries and/or war/conflict-affected areas. This study will contribute to developing a further evidence base for ABPRA that is publicly available and openly accessible. The results would offer invaluable field-informed input to enhance current ABPRA manuals (intervention and research protocols, training manual, and fidelity check sheets). The results would also support Rwandan citizens, organizations, and governments in developing their own programs and policies to furthering reconciliation efforts. Generating further evidence would significantly enhance and improve access to a research-informed interpersonal/psychosocial reconciliation approach to assist the psychosocial reconciliation processes in Rwanda, and potentially other war/conflict-affected areas.

Due to the exploratory nature of this study, our sample size is kept very small $(n=8)$. Consequently, we expect that our quantitative results will be limited in effect size and the potential power to estimate the sample size for the larger-scale evaluation. All other possible limitations to be revealed will be accepted as useful feasibility and acceptability information/data for the future trajectory of the project.

\section{Conclusion}

Development and validation of the PRISM as an outcome measure and generating the field feasibility and acceptability data are essential steps to advance this project to a full-scale experimental evaluation of ABPRA in Rwanda, with the goal to develop ABPRA as an evidence-based post-war/conflict psychosocial reconciliation approach. The product of this project trajectory is a novel intervention developed from the very lived experiences of genocide survivors and perpetrators in a naturalistic sociocultural context of post-genocide Rwanda. It is also evaluated rigorously for the efficacy employing global standards for developing evidencebased complex interventions. In addition to advancing the scholarly efforts in conflict resolution and stimulating further research investigations and collaborations, the proposed work offers the possibility of implementing a scientifically validated and meaningful approach to conflict 
resolution, prevention and peacebuilding to support psychosocial development in war/conflictaffected regions around the globe.

\section{Declarations}

Competing interests: The author declares that he has no competing interests.

Funding: This research is funded by the Social Sciences and Humanities Research Council of Canada

\section{References}

Allport, G. W. (1954). The nature of prejudice. Doubleday Anchor.

Amir, Y. (1969). Contact hypothesis in ethnic relations. Psychological Bulletin, 71(5), 319-342. https://doi.org/10.1037/h0027352

Bar-Tal, D. (2000). From intractable conflict through conflict resolution to reconciliation: Psychological analysis. Political Psychology, 21(2), 351-365. https://doi.org/10.1111/0162-895X.00192

Brewer, M. B., \& Kramer, R. M. (1985). The psychology of intergroup attitudes and behavior. Annual Review of Psychology, 36, 219-243. https://doi.org/10.1146/annurev.ps.36.020185.001251

Cattell R. B. (1966). The scree test for the number of factors. Multivariate Behavioral Research, 1(2), 245-276. https://doi.org/10.1207/s15327906mbr0102_10

Chauvin, L., Mugaju, J., \& Comlavi, J. (1998). Evaluation of the psychosocial trauma recovery programme in Rwanda. Evaluation and Program Planning, 21(4), 385-392. https://doi.org/10.1016/S0149-7189(98)00034-2

Clark, L. A., \& Watson, D. (1998). Constructing validity: Basic issues in objective scale development. In A. E. Kazdin (Ed.), Methodological issues and strategies in clinical research (2nd ed., pp. 215-239). American Psychological Association.

Cliff, N. (1988). The eigenvalues-greater-than-one rule and the reliability of components. Psychological Bulletin, 103(2), 276-279. https://doi.org/10.1037/0033-2909.103.2.276

Craig, P., Dieppe, P., Macintyre, S., Michie, S., Nazareth, I., \& Petticrew, I. (2006). Developing and evaluating complex interventions: New guidance. Retrieved from https://www.mrc.ac.uk/documents/pdf/complex-interventions-guidance/

Cronbach, L. J. (1971). Test validation. In R. L. Thorndike (Ed.), Educational measurement (2nd ed., pp. 443-507). American Council on Education.

Cronbach, L. J. (1951). Coefficient alpha and the internal structure of tests. Psychometrika, 16, 297-334. https://doi.org/10.1007/BF02310555

Cronbach, L. J., \& Meehl, P. E. (1955). Construct validity in psychological tests. Psychological Bulletin, 52(4), 281-302. https://doi.org/10.1037/h0040957

Crotty, M. (1998). The foundations of social research: Meaning and perspective in the research process. SAGE.

DeVellis, R. (2017). Scale development: theory and applications (4th ed.). SAGE.

Dyregrov, A., Gupta, L., Gjestad, R., \& Mukanoheli, E. (2000). Trauma exposure and psychological reactions to genocide among Rwandan children. Journal of Traumatic Stress, 13(1), 3-21. https://doi.org/10.1023/A:1007759112499

Social Science Protocols, February 2021, 1-12.

http://dx.doi.org/10.7565/ssp.v4.5199 
Floyd, F. J., \& Widaman, K. F. (1995). Factor analysis in the development and refinement of clinical assessment instruments. Psychological Assessment, 7(3), 286-299. https://doi.org/10.1037/1040-3590.7.3.286

Furr, R. M., \& Bacharach, V. R. (2014). Psychometrics: An introduction (2 $2^{\text {nd }}$ ed.). SAGE.

Gergen, K. (2001). Social construction in context. SAGE.

Gorsuch, R. L. (1983), Factor analysis (2 ${ }^{\text {nd }}$ ed.). Erlbaum.

Gorsuch, R. L. (2014). Factor analysis: Classic edition. Taylor \& Francis.

Hamber, B. (2007). Forgiveness and reconciliation: Paradise lost or pragmatism? Peace and Conflict: Journal of Peace Psychology, 13(1), 115-125. https://doi.org/10.1037/h0094027

Hinson, L. W. (Director \& Producer). (2009). As we forgive [Documentary]. Sound Entreprises.

Humphreys, L., \& Ilgen, D. R. (1969). Note on a criterion for the number of common factors. Educational and Psychological Measurement, 29, 571 - 578.

Jöreskog, K. G. (1967). Some contributions to maximum likelihood factor analysis. Psychometrika, 32(4), 443-482. https://doi.org/10.1007/BF02289658

Jöreskog, K. G. (1969). A general approach to confirmatory maximum likelihood factor analysis. Psychometrika, 34, 183-202. https://doi.org/10.1007/BF02289343

Krippendorff, K. (2014). Content analysis: An introduction to its methodology (3rd ed.). SAGE. Langdridge, D. (2007). Phenomenological psychology: Theory, research, and method. Pearson. Long, J. S. (1983), Confirmatory factor analysis. SAGE.

McGarty, C. (2014). Twenty years after genocide: The role of psychology in the reconciliation and reconstruction process in Rwanda. Journal of Social and Political Psychology, 2(1), 377386. https://doi.org/10.5964/jspp.v2i1.449

McIntire, S. A., \& Miller, S. A. (2000). Foundations of psychological testing. McGraw-Hill.

Messick, S. (1989). Validity. In R. Linn (Ed.), Educational measurement, (3rd ed., pp. 13-104). American Council on Education.

Minami, M. (2014). Development and field testing of action-based psychosocial reconciliation approach in post-genocide Rwanda (Doctoral dissertation). University of British Columbia. Retrieved from https://open.library.ubc.ca/cIRcle/collections/ubctheses/24/items/1.0167231

Minami, M. (2020). Ubwiyunge mubikorwa (reconciliation in action): Development and field piloting of action-based psychosocial reconciliation approach in post-Gacaca Rwanda. Intervention, 18(2), 129-138.

Morita, S. (1974). Morita Shoma zenshu dai nikan [The standard edition of the complete collected works of Shoma Morita, Vol. 2]. Hakuyosha.

Murphy, K. R., \& Davidshofer, C. O. (1998). Psychological testing: Principles and applications. Prentice Hall.

National Unity and Reconciliation Commission. (2020). National policy of unity and reconciliation. Retrieved from https://www.nurc.gov.rw/fileadmin/Documents/Others/National_Policy_of_Unity_and_Recon ciliation.pdf

Netemeyer, R. G., Bearden, W. O., \& Sharma, S. (2003). Scaling procedures: Issues and applications. SAGE.

Nunnally, J. C., \& Bernstein, I. H. (1994). Psychometric theory. McGraw-Hill.

Pedhazur, E. J., \& Schmelkin, L. P. (1991). Measurement, design, and analysis: An integrated approach. Lawrence Erlbaum Associates.

Pett, M. A., Lackey, N. R., \& Sullivan, J. J. (2003). Making sense of factor analysis: The use of factor analysis for instrument development in health care research. SAGE.

Social Science Protocols, February 2021, 1-12.

http://dx.doi.org/10.7565/ssp.v4.5199 
Pettigrew, T. F. (1997). Generalized intergroup contact effects on prejudice. Personality and Social Psychology Bulletin, 23(2), 173-185. https://doi.org/10.1177/0146167297232006

Pettigrew, T. F. (1998). Intergroup contact theory. Annual Review of Psychology, 49, 65-85. https://doi.org/10.1146/annurev.psych.49.1.65

Pettigrew, T. F., \& Tropp, L. R. (2006). A meta-analytic test of intergroup contact theory. Journal of Personality and Social Psychology, 90(5), 751-783. https://doi.org/10.1037/0022-3514.90.5.751

Smith, J. A., Flowers, P., \& Larkin, M. (2009). Interpretative phenomenological analysis: Theory, method and research. SAGE.

Spector, P. E. (1992). Summated rating scale construction: An introduction. SAGE.

Stammel, N., Bockers, E., Neuner, F., Chhim, S., Taing, S., \& Knaevelsrud, C. (2015). The readiness to reconcile inventory. European Journal of Psychological Assessment, 33(6), 436444. http://doi.org/10.1027/1015-5759/a000304

Staub, E. (2008). Promoting reconciliation after genocide and mass killing in Rwanda-And other postconflict settings: Understanding the roots of violence, healing, shared history, and general principles. In A. Nadler, T. E. Malloy, \& J. D. Fisher (Eds.), The social psychology of intergroup reconciliation (p. 395-422). Oxford University Press. https://doi.org/10.1093/acprof:oso/9780195300314.003.0018

Staub, E., Pearlman, L. A., Gubin, A., \& Hagengimana, A. (2005). Healing, reconciliation, forgiving and the prevention of violence after genocide or mass killing: An intervention and its experimental evaluation in Rwanda. Journal of Social and Clinical Psychology, 24, 297334.

Thabane, L., Ma, J., Chu, R., Cheng, J., Ismaila, A., Rios, L. P., Robson, R., Thabane, M., Giangregorio, L., \& Goldsmith, C. H. (2010). A tutorial on pilot studies: The what, why and how. BMC Medical Research Methodology, 10(1). https://doi.org/10.1186/1471-2288-10-1

van Manen, M. (2016). Researching lived experience: Human science for an action sensitive pedagogy. Taylor \& Francis.

Worthington, E. L., Jr. (2005). Handbook of forgiveness. Brunner/Routledge.

Worthington, E. L., Jr. (2006). Forgiveness and reconciliation: Theory and application. Brunner/Routledge. 